\title{
Minimal detectable biases of GPS data
}

\author{
P. J. G. Teunissen \\ Delft Geodetic Computing Centre (LGR), Faculty of Geodesy, Delft University of Technology, \\ Thijsseweg 11, NL-2629 JA Delft, The Netherlands \\ Fax: + 3115278 3711; e-mail: P.J.G.Teunissen@geo.tudelft.nl
}

Received: 23 April 1997 / Accepted: 27 October 1997

\begin{abstract}
In this contribution closed-form expressions are given for the minimal detectable biases of single- and dual-frequency pseudo-range and carrier-phase data. They are given for three different single-baseline models. These are the geometry-free model and two variants of the geometry-based model, namely the roving and stationary variants. The baselines are considered to be sufficiently short such that orbital uncertainties in the fixed orbits and residual ionospheric and tropospheric delays can be assumed absent. The stochastic model used is one that permits cross-correlation and the use of different variances for individual GPS observables, including the possibility to weigh the observables in dependence on which satellite is tracked.
\end{abstract}

Key words. GPS · MDB · Outliers · Cycle slips

\section{Introduction}

Minimal detectable biases (MDBs) as introduced by Baarda $(1967,1968)$ are an important diagnostic tool for inferring the strength of model validation. Within the context of GPS the model used typically depends on the particular application at hand. There is a whole suite of GPS models to consider. A GPS model for relative positioning may be based on the simultaneous use of two receivers (single-baseline) or more than two receivers (multi-baseline or network). It may have the relative receiver-satellite geometry included (geometry-based) or excluded (geometry-free). When geometry is excluded, the baseline components are not involved as unknowns in the model, but instead, the receiver-satellite ranges themselves. GPS models may also be discriminated as to whether the slave receiver(s) are in motion (nonstationary) or not (stationary). When in motion, one solves for one or more trajectories, since with the receiver-satellite geometry included, one will have a new baseline for each new epoch.
The fact that a whole suite of different GPS models exists implies that there are different stages at which quality control can be exercised. Roughly speaking, one can discriminate between the following four levels:

1. Receiver-level: in principle it is already possible to validate the time-series of undifferenced data of a single receiver. By lumping some of the parameters (e.g. range, clock errors, tropospheric delay, orbital uncertainty) and by introducing a smoothness constraint on the time behaviour of the ionospheric delays, redundancy enters which can be used for validation. Single-receiver quality control is very useful for reference receivers that are used in active GPS control networks or in DGPS, see e.g. Jin et al. (1995), Jin (1995) and Jong (1996, 1997).

2. Baseline-level: in this case the observation equations are parametrized in terms of the baseline vector of the two receivers. Here the redundancy primarily stems from the presence in the design matrix of the receiversatellite geometry and from the assumed constancy over time of the ambiguities. Additional redundancy enters when the baseline is considered stationary instead of moving.

3. Network-level: when sufficient (independent) baselines are used to form a network, redundancy enters by enforcing the closure of 'baseline loops'. The redundancy characteristics of a baseline network are very similar to that of a classical levelling network. The quality control is therefore not unlike that of the terrestrial networks, see e.g. Teunissen (1985), Marel (1990) and Leick (1995).

4. Connection-level: additional redundancy enters again when a free GPS network is connected to points of an existing geodetic control. In this case the redundancy stems from the fact that the shape of the free network is compared with the shape of the existing control network, see e.g. LGR staff (1982), Teunissen and Verhoef (1996).

In this contribution we consider models of the singlebaseline type, with receivers separated by a short distance only. The term 'short' refers to the assumption 
that the double-differenced GPS observables are sufficiently insensitive to orbital uncertainties in the fixed orbits and to residual ionospheric and tropospheric delays. Within this class, three different single-baseline models will be considered. They are the geometry-free model and two variants of the geometry-based model, namely the roving variant and the stationary variant.

The geometry-free model is the simplest single-baseline model to consider. Since it dispenses with the receiver-satellite geometry, pseudo-range (code) data are required to solve for all the unknown parameters. The use of the geometry-free model has been studied by many (e.g. Hatch 1982; Euler and Goad 1991; Dedes and Goad 1994; Euler and Hatch 1994; Teunissen 1996). Both variants of the geometry-based model make explicit use of the receiver-satellite geometry. The only difference between the two variants is whether or not the baseline is assumed stationary over the observation time-span. The use of the geometry-based model also has been studied by many - Frei and Beutler (1990), Hatch (1991), Teunissen et al. (1995) and Tiberius and de Jonge (1995) to mention just a few.

This contribution is organized as follows. In Sect. 2 a brief review is given of the concept of internal reliability. In Sect. 3 the three single-baseline models are introduced and a compact way of describing their functional and stochastic models is provided. Based on the particular structure of each of the three single-baseline models, an orthogonal decomposition is given of the least-squares projector in Sect. 4. The least-squares projector is needed for deriving the MDBs and the orthogonal decomposition facilitates this computation. In Sects. 5 and 6 the closed-form expressions for the MDBs are given. In Sect. 5 outliers in the code data are considered and in Sect. 6 cycle slips in the phase data are studied.

The following notation will be used throughout. The identity matrix of order $p$ will be denoted by $I_{p}$, the $p$ vector having all 1's as its entries by $e_{p}$ and the canonical unit vector having 1 as its $p$ th entry by $c_{p}$. The Kronecker product is denoted by $\otimes$ and the capital $P$ is reserved for projectors. The Kronecker product of two matrices $M$ and $N$ is defined as

$M \otimes N=\left[\begin{array}{ccc}m_{11} N & \ldots & m_{1 q} N \\ \vdots & & \vdots \\ m_{p 1} N & \ldots & m_{p q} N\end{array}\right]$

with $M=\left[m_{i j}\right], i=1, \ldots, p, j=1, \ldots, q$; some of its properties are (Rao 1973)

$$
\begin{gathered}
(M \otimes N)^{T}=M^{T} \otimes N^{T} \\
(M \otimes N)^{-1}=M^{-1} \otimes N^{-1} \\
\left(M_{1} \otimes N_{1}\right)\left(M_{2} \otimes N_{2}\right)=M_{1} M_{2} \otimes N_{1} N_{2}
\end{gathered}
$$

Extensive use of orthogonal projectors will also be made; some of their properties are:

$$
\begin{aligned}
& P_{M}=M\left[M^{T} Q^{-1} M\right]^{-1} M^{T} Q^{-1} \\
& P_{M}^{\perp}=I-P_{M} \\
& P_{M}=P_{M X} \text { if } X \text { invertible }
\end{aligned}
$$

$$
\begin{aligned}
& P_{M}=P_{M_{1}}+P_{P_{M_{1}}^{\perp} M_{2}} \text { if } M=\left[M_{1}, M_{2}\right] \\
& P_{M}=P_{M_{1}} \otimes P_{M_{2}} \text { if } M=M_{1} \otimes M_{2}, Q=Q_{1} \otimes Q_{2}
\end{aligned}
$$

The first equality of Eq. (2) defines the orthogonal projector $P_{M}$. It projects onto the range space of matrix $M$ and along its orthogonal complement. Orthogonality is taken here with respect to the metric $Q^{-1}$. Equation (2b) defines the complementary projector of $P_{M}$. The complementary projector $P_{M}^{\perp}$ projects onto the orthogonal complement of $M$ and along the range space of $M$. The third equality of Eq. (2) shows that projectors are independent of the choice of parametrization. That is, they are uniquely characterized by the spaces onto which they project and thus not dependent on the way these spaces are represented. Equation (2d) shows the orthogonal decomposition of a projector, while Eq. (2e) shows that the projection onto a Kronecker product equals the Kronecker product of the projections provided the metric is a Kronecker product as well.

\section{Internal reliability}

The concept of reliability is an important diagnostic tool for inferring the strength with which mathematical models can be validated, cf. Baarda (1968), Teunissen (1985) and Marel (1990). Internal reliability as represented by the MDB describes the size of the model errors which can just be detected with the appropriate test statistics.

Let our null hypothesis $H_{0}$ be specified as

$H_{0}: E\{y\}=A x, D\{y\}=Q_{y}$

where $E\{$.$\} and D\{$.$\} are, respectively, the expectation$ and dispersion operator, $y$ is the $m$-vector of normally distributed observables, $A$ is the $m \times n$ design matrix, $x$ is the $n$-vector of unknown parameters and $Q_{y}$ is the variance matrix of the observables. We assume the alternative hypothesis $H_{a}$ to be a mean-shifted version of the null hypothesis

$H_{a}: \quad E\{y\}=A x+b_{y}, D\{y\}=Q_{y}$

where the bias vector $b_{y}$ describes the model error. In this contribution it is assumed that the model error is one-dimensional; that is, the bias $b_{y}$ can be parametrized by means of a single parameter $\nabla$ as $b_{y}=c \nabla$, where the $m$-vector $c$ is assumed known and the scalar $\nabla$ unknown. The vector $c$ specifies the type of model error. The uniformly most powerful test statistic for testing $H_{0}$ against $H_{a}$ is given as

$T=\frac{\left(c^{T} Q_{y}^{-1} P_{A}^{\perp} y\right)^{2}}{c^{T} Q_{y}^{-1} P_{A}^{\perp} c}$

where $P_{A}^{\perp}=I_{m}-P_{A}$ and $P_{A}$ is the least-squares projector. The least-squares projector projects onto the range space of $A$ and along its orthogonal complement. The test statistic $T$ has the following Chi-squared distributions under $H_{0}$ and $H_{a}$

$H_{0}: T \sim \chi^{2}(1,0) ; H_{a}: T \sim \chi^{2}(1, \lambda)$ 
with the non-centrality parameter

$\lambda=\nabla^{2} c^{T} Q_{y}^{-1} P_{A}^{\perp} c$

The non-centrality parameter can be computed once the level of significance (probability of type-I error) and the detection power ( 1 minus probability of type-II error) are chosen. A level of significance of $\alpha_{0}$ together with a power of $\gamma_{0}$ will give a non-centrality parameter of $\lambda_{0}=$ $\lambda\left(\alpha_{0}, \gamma_{0}\right)$. For instance, for $\alpha_{0}=0.001$ and $\gamma_{0}=0.80$, it follows that $\lambda_{0} \approx 17$. Once the non-centrality parameter is known, the quadratic equation (7) can be inverted to obtain the corresponding size of the bias

$|\nabla|=\sqrt{\frac{\lambda_{0}}{c^{T} Q_{y}^{-1} P_{A}^{\perp} c}}$

This is the celebrated MDB. It is the minimal size of the bias that can be detected with the test statistic $T$ when the level of significance and the power are set at, respectively, $\alpha_{0}$ and $\gamma_{0}$. Apart from the chosen level of significance and the power, the MDB only depends on the vector $c$, the design matrix $A$ and the variance matrix $Q_{y}$. Since $c$ specifies the type of model error, different model errors will have different MDBs. In this contribution outliers in the pseudo-range (code) data and cycle slips in the phase data will be considered as model error. But before their MDBs can be computed, one first needs to know the structure of the design matrix $A$. In the next section this design matrix will be given for three different GPS single-baseline models.

\section{Three single-baseline models}

When tracking satellites $r$ and $s$ at epoch $t$ using two receivers $i$ and $j$ that are not too far apart, the doubledifferenced (DD) observation equations for singlefrequency code and phase read (cf. Hofmann-Wellenhof et al. 1997; Leick 1995; Teunissen and Kleusberg 1996):

$$
\begin{aligned}
& p_{i j}^{r s}(t)=\rho_{i j}^{r s}(t)+n_{p, i j}^{r s}(t) \\
& \phi_{i j}^{r s}(t)=\rho_{i j}^{r s}(t)+\lambda N_{i j}^{r s}+n_{\phi, i j}^{r s}(t)
\end{aligned}
$$

where $p_{i j}^{r s}(t)$ is the DD code observable, $\phi_{i j}^{r s}(t)$ is the DD phase observable expressed in range units rather than cycles, $\rho_{i j}^{r s}(t)$ is the unknown DD receiver-satellite range, $N_{i j}^{r s}$ is the integer carrier-phase ambiguity that corresponds with the wavelength $\lambda$, and $n_{\phi, i j}^{r s}(t)$ and $n_{p, i j}^{r s}(t)$ are, respectively, the DD noises of phase and code.

With dual-frequency data there is one such pair of equations for each of the two frequencies $L_{1}$ and $L_{2}$. Thus when $m$ satellites are tracked there are $2(m-1)$ measurements per frequency. Their model equations can be written in vector form as

$$
\begin{aligned}
& p_{1}(t)=\rho(t)+n_{p_{1}}(t) \\
& p_{2}(t)=\rho(t)+n_{p_{2}}(t) \\
& \phi_{1}(t)=\rho(t)+\lambda_{1} N_{1}+n_{\phi_{1}}(t) \\
& \phi_{2}(t)=\rho(t)+\lambda_{2} N_{2}+n_{\phi_{2}}(t)
\end{aligned}
$$

where the indices for the two receivers and the $m$ satellites have been omitted. The lower index now refers to one of the two frequencies. Using the notation $y_{k}=\left(p_{1}\left(t_{k}\right)^{T}, p_{2}\left(t_{k}\right)^{T}, \phi_{1}\left(t_{k}\right)^{T}, \phi_{2}\left(t_{k}\right)^{T}\right)^{T}, \rho_{k}=\rho\left(t_{k}\right), a=$ $\left(\lambda_{1} N_{1}^{T}, \lambda_{2} N_{2}^{T}\right)^{T}$ and $n_{k}=\left(n_{p_{1}}\left(t_{k}\right)^{T}, n_{p_{2}}\left(t_{k}\right)^{T}, n_{\phi_{1}}\left(t_{k}\right)^{T}\right.$, $\left.n_{\phi_{2}}\left(t_{k}\right)^{T}\right)^{T}$, these equations can be written in a more compact form as

$y_{k}=\left(e_{4} \otimes I_{m-1}\right) \rho_{k}+\left(C_{2} \otimes I_{m-1}\right) a+n_{k}$ with $C_{2}=c_{2} \otimes I_{2}$

With this result we are now in a position to formulate the complete set of observation equations for the three single-baseline models.

\subsection{Geometry-free model}

In this model the observation equations are not parametrized in terms of the baseline components. Instead, they remain parametrized in terms of the unknown DD receiver-satellite ranges. This implies that the observation equations remain linear and that the receiver-satellite geometry is not explicitly present in these equations. Hence the model permits both receivers to be either stationary or moving. Since the equations as given by Eq. (11) are the ones that belong to the geometry-free model, we get for $k$ epochs of data

$y=\left[I_{k} \otimes\left(e_{4} \otimes I_{m-1}\right)\right] \rho+\left[e_{k} \otimes\left(C_{2} \otimes I_{m-1}\right)\right] a+n$

where for $i=1, \ldots, k$, the $y_{i}, \rho_{i}$ and $n_{i}$ are collected in, respectively, $y, \rho$ and $n$. The redundancy of the model equals $(m-1)(3 k-2)$ for the dual-frequency case and $(m-1)(k-1)$ for the single-frequency case. Thus in order to have redundancy, we need to track two or more satellites while using at least one epoch for the dualfrequency case or at least two epochs for the singlefrequency case.

\subsection{Roving-receiver geometry-based model}

In case of the geometry-based model, the observation equations are parametrized in terms of the baseline components. As a consequence the relative receiversatellite geometry enters the model. Since the equations are non-linear, a linearization of the DD receiversatellite ranges with respect to the baseline components is needed

$\Delta \rho_{k}=\bar{G}_{k} \Delta b_{k}$ with $\bar{G}_{k}=\left[\frac{\partial \rho_{k}}{\partial b_{k}}\right]$

where $b_{k}$ denotes the baseline vector of epoch $k$ and $\Delta b_{k}$ its increment. The geometry of the DD relative receiversatellite configuration is captured in the $(m-1) \times 3$ matrix $\bar{G}_{k}$.

It is well known that due to the high-altitude orbits of the GPS satellites, the receiver-satellite geometry changes only slowly with time. The matrix $\bar{G}_{k}$ is therefore only weakly dependent on time. In our further analysis 
it will therefore be assumed that $\bar{G}_{k}$ is a time-invariant matrix, $\bar{G}_{k}=\bar{G}=$ constant. This approximation is allowed for short time-spans, in particular since our attention is restricted to the computation of the MDBs only. With $\bar{G}_{k}=\bar{G}$ and $b=\left(b_{1}^{T}, \ldots, b_{k}^{T}\right)^{T}$, it follows from Eq. (13) that

$\rho=\left(I_{k} \otimes \bar{G}\right) b$

where the $\Delta$-symbol has been omitted for notational convenience. From substituting Eq. (14) into Eq. (12), the DD observation equations follow as

$y=\left[I_{k} \otimes\left(e_{4} \otimes \bar{G}\right)\right] b+\left[e_{k} \otimes\left(C_{2} \otimes I_{m-1}\right)\right] a+n$

For the single-frequency case, the redundancy of the model equals $(m-1)(2 k-1)-3 k$. Thus in order to have redundancy for a single epoch, more than four satellites need to be tracked. For the dual-frequency case, the redundancy equals $2(m-1)(2 k-1)-3 k$. For a single epoch this gives $2 m-5$. Due to the fact that redundancy may still exist when the design matrix is rank defect, one should be aware of the following. One should not conclude that for $k=1$ redundancy only exists when $m \geq 3$; it already exists for $m \geq 2$. This is due to the presence of the dual-frequency code data. Thus for $k=1$, the redundancy equals $(m-1)$ when $m \leq 4$ and equals $(2 m-5)$ when $m \geq 4$. The dual-frequency redundancy $2(m-1)(2 k-1)-3 k$ therefore holds true under the assumption that the baseline components are estimable.

Note that the geometry-free model follows from that of the roving-receiver geometry-based model when the matrix $\bar{G}$ is replaced by the identity matrix $I_{m-1}$ and $b$ is replaced by $\rho$. This implies that the geometry-free model is just a special case of the roving variant.

\subsection{Stationary-receiver geometry-based model}

When the two receivers are stationary, the $k$ baselines $b_{k}$ collapse to one single baseline $b^{*}$. We therefore have

$b=\left(e_{k} \otimes I_{3}\right) b^{*}$

The corresponding DD observation equations follow then from substituting Eq. (16) into Eq. (15) as

$y=\left[e_{k} \otimes\left(e_{4} \otimes \bar{G}\right)\right] b^{*}+\left[e_{k} \otimes\left(C_{2} \otimes I_{m-1}\right)\right] a+n$

The single-frequency and dual-frequency redundancies now equal, respectively, $(m-1)(2 k-1)-3$ and $2(m-1)(2 k-1)-3$. When compared to the previous model the redundancy has increased by $3(k-1)$, which equals the number of baseline components that have been constrained.

\subsection{Stochastic model}

In order to compute the MDBs one needs, apart from the functional model, also the stochastic model. It will be clear that the stochastic model cannot be chosen arbitrarily. A proper stochastic model should reflect the uncertainty which is assumed present in the a priori residuals. For the present contribution a rather general stochastic model is taken. The variance matrix of the observables is assumed to be given as

$Q_{y}=I_{k} \otimes Q$ with $Q=C_{p \phi} \otimes E$

where

$C_{p \phi}=\operatorname{blockdiag}\left(C_{p}, C_{\phi}\right)$ and $E=D^{T} W^{-1} D$

with the $2 \times 2$ single-differenced (SD) cofactor matrices

$C_{p}=\left[\begin{array}{cc}c_{p_{1}}^{2} & c_{p_{1} p_{2}} \\ c_{p_{2} p_{1}} & c_{p_{2}}^{2}\end{array}\right], C_{\phi}=\left[\begin{array}{cc}c_{\phi_{1}}^{2} & c_{\phi_{1} \phi_{2}} \\ c_{\phi_{2} \phi_{1}} & c_{\phi_{2}}^{2}\end{array}\right]$

and the diagonal weight matrix $W=\operatorname{diag}\left(w_{1}, \ldots, w_{m}\right)$. The $(m-1) \times m$ matrix $D^{T}$ is the operator that transforms single-differences into double-differences.

Through the two cofactor matrices $C_{\phi}$ and $C_{p}$ the variances of the observables are allowed to differ on the two frequencies. Also, the presence of cross-correlation is permitted. Depending on how the measurement process is implemented in the GPS receivers, the observables may or may not be cross-correlated. In the presence of anti-spoofing (AS) for instance, some receivers use a hybrid technique to provide dual-frequency code measurements (Hofmann-Wellenhof et al. 1997). As a result the code data become cross-correlated.

The $(m-1) \times(m-1)$ matrix $E$ can be used to weigh the observables individually depending on which satellite is tracked. It can be used for instance to include a satellite elevation dependency. Satellite elevation dependency of the precision of the GPS observables has been studied by Euler and Goad (1991) and Jin (1995). Strictly speaking, the matrix $E$ should then be time dependent as well. For short time-spans, however, it can be taken as a constant matrix due to the slowly changing GPS receiver-satellite geometry.

\section{Decomposing the least-squares projector}

Now that the structure of the three single-baseline models is known, one can start to construct the leastsquares projector $P_{A}$, needed for computing the MDBs. The design matrix $A$ follows from the results of the previous section as

$A=\left[M_{k}, N_{k}\right]$ with $M_{k}=I_{k} \otimes M, N_{k}=e_{k} \otimes N$

where

$\left\{\begin{array}{lll}\text { geometry-free: } & M=e_{4} \otimes I_{m-1} \quad N=C_{2} \otimes I_{m-1} \\ \text { roving-receiver: } & M=e_{4} \otimes \bar{G} \quad N=C_{2} \otimes I_{m-1} \\ \text { stationary-receiver: } & M=0 \quad N=\left(e_{4} \otimes \bar{G}, C_{2} \otimes I_{m-1}\right)\end{array}\right.$

Let us now decompose the least-squares projector stepby-step in order to obtain a form which is suited for a direct computation of the MDBs. For that purpose extensive use will be made of the projector properties in Eq. (2). From Eqs. (18) and (19) it follows that 


$$
\begin{aligned}
P_{\left[M_{k}, N_{k}\right]} & =P_{M_{k}}+P_{P_{M_{k}}^{\perp} N_{k}}, & & P_{M_{k}}^{\perp} N_{k}=e_{k} \otimes P_{M}^{\perp} N \\
P_{M_{k}} & =I_{k} \otimes P_{M}, & & P_{P_{M_{k}}^{\perp} N_{k}}=P_{e_{k}} \otimes P_{P_{M}^{\perp} N} \\
P_{M_{k}}^{\perp} & =I_{k} \otimes P_{M}^{\perp}, & & P_{[M, N]}=P_{M}+P_{P_{M}^{\perp} N}^{\perp}
\end{aligned}
$$

This shows, since $P_{A}=P_{\left[M_{k}, N_{k}\right]}$, that the least-squares projector can be decomposed into a projector sum of two Kronecker products

$$
P_{A}=P_{e_{k}}^{\perp} \otimes P_{M}+P_{e_{k}} \otimes P_{[M, N]}
$$

This decomposition clearly shows how the time-dependent and the time-invariant entries of the design matrix contribute to the projector. Also note that the decomposition is valid for all three single-baseline models. Although the $M$ and $N$ matrices differ for the rovingreceiver and the stationary-receiver cases, the range space of the matrix $[M, N]$ does not. This implies that the second term in the sum of Eq. (20) will be the same for both models. Thus one only needs to set $P_{M}=0$ to obtain the stationary-receiver result from the rovingreceiver result. And from the roving-receiver result one can also obtain the geometry-free result, simply by replacing matrix $\bar{G}$ with $I_{m-1}$.

A further decomposition is possible if one considers the two projectors $P_{M}$ and $P_{[M, N]}$ for the case of the roving receiver. From Eqs. (18) and (19) it follows that

$P_{M}=P_{e_{4}} \otimes P_{\bar{G}}$ and $P_{[M, N]}=P_{C_{1}} \otimes P_{\bar{G}}+P_{C_{2}} \otimes I_{m-1}$

with $C_{1}=c_{1} \otimes e_{2}$ and $C_{2}=c_{2} \otimes I_{2}$. Since $C_{1}$ relates to the code data and $C_{2}$ to the phase data, the decomposition of $P_{[M, N]}$ has the property that the first term in the sum vanishes when cycle slips are considered, while the second term vanishes when outliers in the code data are considered. With Eqs. (20) and (21) the minimal detectable biases can be derived. First let us consider outliers in the code data, then cycle slips in the phase data.

\section{The outlier MDBs}

In this section outliers in the code data are considered. The outlier MDBs will be derived for all three singlebaseline models. In order to compute the MDB one first needs to specify the appropriate $c$-vector. For an $L_{1}$ code outlier at epoch $l(1 \leq l \leq k)$ in the range to satellite $i \in\{1, \ldots, m\}$, the $c$-vector takes the form

$c=c_{l} \otimes d$ with $d=c_{1} \otimes d_{i}$

The vector $c_{l}$ picks the data of epoch $l$, while the vector $c_{1}$ selects the $L_{1}$ code data of that epoch. The vector $d_{i}$ describes how a range error to satellite $i$ affects the DD range vector. Since $c_{l}^{T} P_{e_{k}} c_{l}=\frac{1}{k}$, the general expression for the outlier MDB follows from Eqs. (8), (18), (20) and (22) as

$$
\left|\nabla_{p_{1}}\right|=\sqrt{\frac{\lambda_{0} / d^{T} Q^{-1} d}{1-\left(1-\frac{1}{k}\right) \frac{d^{T} Q^{-1} P_{M} d}{d^{T} Q^{-1} d}-\frac{1}{k} \frac{d^{T} Q^{-1} P_{[M, N]} d}{d^{T} Q^{-1} d}}}
$$

This expression still holds true for all three singlebaseline models. Its entries can be broken down into smaller parts if Eq. (21) is used. For the roving-receiver case this gives

$$
\begin{aligned}
d^{T} Q^{-1} d & =d_{i}^{T} E^{-1} d_{i} / w_{i} \sigma_{p_{1}^{i} \mid p_{2}^{i}}^{2} \\
\frac{d^{T} Q^{-1} P_{M} d}{d^{T} Q^{-1} d} & =\delta_{p_{1}} \frac{\epsilon}{1+\epsilon} \frac{d_{i}^{T} E^{-1} P_{\bar{G}} d_{i}}{d_{i}^{T} E^{-1} d_{i}} \\
\frac{d^{T} Q^{-1} P_{[M, N]} d}{d^{T} Q^{-1} d} & =\delta_{p_{1}} \frac{d_{i}^{T} E^{-1} P_{\bar{G}} d_{i}}{d_{i}^{T} E^{-1} d_{i}}
\end{aligned}
$$

with

$$
\begin{aligned}
& \delta_{p_{1}}=1-\frac{\sigma_{\bar{p}}^{2}}{\sigma_{p_{2}}^{2}}, \quad \epsilon=\frac{\sigma_{\bar{\phi}}^{2}}{\sigma_{\bar{p}}^{2}} \text { and } \\
& \sigma_{p_{1}^{i} \mid p_{2}^{i}}^{2}=\frac{1}{w_{i}}\left(c_{p_{1}}^{2}-c_{p_{1} p_{2}} c_{p_{2}}^{-2} c_{p_{2} p_{1}}\right)
\end{aligned}
$$

where $\sigma_{\bar{p}}^{2}$ and $\sigma_{\bar{\phi}}^{2}$ are the SD variances of the weighted mean of, respectively, the $L_{1}$ and $L_{2}$ code observables and the $L_{1}$ and $L_{2}$ phase observables, and where $\sigma_{p_{1}^{i} \mid p_{2}^{i}}^{2}$ is the SD conditional variance of the $L_{1}$ code observable for satellite $i$. Note that both $d_{i}$ and $E$ still refer to DD variates. Since this obscures the interpretation of Eq. (23) somewhat, a representation in terms of SD variates is desired. It follows that

$$
\begin{aligned}
d_{i}^{T} E^{-1} d_{i} & =c_{i}^{T} W P_{e_{m}}^{\perp} c_{i} \\
d_{i}^{T} E^{-1} P_{\bar{G}} d_{i} & =c_{i}^{T} W P_{e_{m}}^{\perp} c_{i}-c_{i}^{T} W P_{\left[G, e_{m}\right]}^{\perp} c_{i}
\end{aligned}
$$

where the $m \times 3$ matrix $G$ is the SD version of $\bar{G}$. Thus $\bar{G}=D^{T} G$.

To prove the first part of Eq. (25), recall that vector $d_{i}$ describes the DD impact of a range error to satellite $i$. Thus $d_{i}=D^{T} c_{i}$. Combined with $E=D^{T} W^{-1} D$, it gives $d_{i}^{T} E^{-1} d_{i}=c_{i}^{T} D\left[D^{T} W^{-1} D\right]^{-1} D c_{i}$. The result now follows from using the projector identity $P_{e_{m}}^{\perp}=I_{m}-$ $e_{m}\left[e_{m}^{T} W e_{m}\right]^{-1} e_{m}^{T} W=W^{-1} D\left[D^{T} W^{-1} D\right]^{-1} D^{T}$. This identity holds true, since $D^{T} e_{m}=0$ and the range space of $D$ is complementary to that of $e_{m}$. The second part of Eq. (25) follows by substituting $d_{i}=D^{T} c_{i}, P_{\bar{G}} D^{T}=D^{T} P_{P_{e_{m}} G}$ and $P_{\left[G, e_{m}\right]}^{\perp}=P_{e_{m}}^{\perp}-P_{P_{e_{m}} G}$. Finally note that the first part of Eq. (25) is only determined by the weights $w_{i}$, $i=1, \ldots, m$. We have

$c_{i}^{T} W P_{e_{m}}^{\perp} c_{i}=w_{i}\left[1-\frac{w_{i}}{\sum_{j=1}^{m} w_{j}}\right]$

Consideration of each of the three single-baseline models individually is now possible.

\subsection{Geometry-free model}

In this case the receiver-satellite geometry is absent. Hence $\bar{G}$ is replaced with $I_{m-1}$. This amounts to replacing the projector $P_{\bar{G}}$ in Eq. (24) by the unit matrix or the projector $P_{\left[G, e_{m}\right]}^{\perp}$ in Eq. (25) by the zero matrix. The outlier MDB follows now from substituting Eqs. (26), (25) and (24) into Eq. (23) as 


$$
\left|\nabla_{p_{1}^{i}}\right|=\sigma_{p_{1}^{i} \mid p_{2}^{i}} \sqrt{\frac{\lambda_{0}}{\left[1-\frac{w_{i}}{\sum_{j=1}^{m} w_{j}}\right]\left[1-\frac{\delta_{p_{1}}}{k}\left(\frac{1+k \epsilon}{1+\epsilon}\right)\right]}}
$$

This is the MDB for the $L_{1}$ code observable. Interchanging $p_{1}$ and $p_{2}$ gives the MDB for the $L_{2}$ code observable. The MDB depends on $\alpha_{o}, \gamma_{o}$, on the precision of code, on the precision of phase, on the number of epochs used and, through the weights $w_{i}$, also on the number of satellites tracked. It is independent, however, of the moment the outlier occurred.

The dependence of the MDB on the number of satellites tracked is due to the DD process involved. Thus the MDB gets smaller when the number of satellites gets larger. The MDB also gets smaller when the weight $w_{i}$ gets larger. Thus in case of satellite elevation dependency, lower satellites produce larger MDBs in which case the internal reliability will be poorer.

The expression given holds for the dual-frequency case. The corresponding expression for the single-frequency case follows by replacing $\sigma_{p_{1}^{i} \mid p_{2}^{i}}$ with $\sigma_{p_{1}^{i}}$ and by setting $\delta_{p_{1}}$ equal to 1 . When cross-correlation is absent and when the $L_{1}$ variance equals the $L_{2}$ variance, we have $\delta_{p_{1}}=0.5$. This shows that the dual-frequency MDB acts as a single-frequency MDB with twice as many epochs. This is also clear if $k=1$. For the singlefrequency case this gives $\delta_{p_{1}}=1$ and therefore $\left|\nabla_{p_{1}^{i}}\right|=\infty$. For the dual-frequency case, however, it gives a finite value for the MDB.

Although the MDB depends on the precision of the phase data, this dependency is only very weakly present in the preceding expression. This is due to the very small value of the phase-code variance ratio $\epsilon$, which in practice is in the order of about $10^{-4}$. This shows that the precision of the phase data has no significant impact on the outlier MDB. In other words, the MDB is predominantly governed by the precision of the code data.

\subsection{Roving receiver}

In the case of a roving receiver the presence of the receiver-satellite geometry is explicitly taken into account. The baselines are non-stationary however. The corresponding outlier MDB follows then from substituting Eqs. (26), (25) and (24) into Eq. (23) as tor $P_{\left[G, e_{m}\right]}$. The two MDBs become identical when $c_{i}^{T} P_{\left[G, e_{m}\right]} c_{i}=1$. This happens in two cases. It happens when the projector is an identity matrix, and it happens when the vector $c_{i}$ lies in the range space of $\left[G, e_{m}\right]$. The first case occurs when there is no satellite redundancy. Then $m=4$ and matrix $\left[G, e_{m}\right]$ will be a square matrix. This matrix will then be invertible in the absence of configuration defects. As a result the projector reduces to an identity matrix.

The second case occurs for certain receiver-satellite configurations. Let matrix $G$ be given as $G=$ $\left[g_{1}, \ldots, g_{m}\right]^{T}$, with $g_{i}$ the direction cosine vector from receiver to satellite $i$. If we assume that a vector $r$ exists such that $g_{i}^{T} r=0$ and $g_{j}^{T} r=-1$ for $j \neq i$, then $G r+e_{m}=c_{i}$, which shows that $c_{i}$ lies in the range space of $\left[G, e_{m}\right]$. Geometrically this translates to a configuration where all satellites except satellite $i$ lie on a cone with axis of symmetry along vector $r$, while satellite $i$ lies in a plane perpendicular to $r$. Note that vector $r$ may have any direction in space. Thus when $r$ points to the local zenith, the configuration would be one where all satellites except satellite $i$ have the same elevation, while satellite $i$ itself has zero elevation.

\subsection{Stationary receiver}

In this case the baseline is assumed stationary. As noted earlier this has as a consequence that the projector $P_{M}$ vanishes. The corresponding outlier MDB follows therefore from substituting Eqs. (26), (25) and (24), with $P_{M}=0$, into Eq. (23) as

$$
\left|\nabla_{p_{1}^{i}}\right|=\sigma_{p_{1}^{i} \mid p_{2}^{i}} \sqrt{\frac{\lambda_{0}}{\left[1-\frac{w_{i}}{\sum_{j=1}^{m} w_{j}}\right]\left[1-\frac{\delta_{p_{1}}}{k}\right]+\frac{\delta_{p_{1}}}{k}\left[1-c_{i}^{T} P_{\left[G, e_{m}\right]} c_{i}\right]}}
$$

This MDB is again smaller than the roving-receiver case. Note, however, that the two MDBs become identical when $\epsilon$ is set to zero. Since the phase-code variance ratio is in fact very small in practice, this shows that the two MDBs will not differ by much. The conclusion is therefore reached that one's ability to detect outliers in the code data is practically independent of whether the baseline is stationary or not.

$$
\left|\nabla_{p_{1}^{i}}\right|=\sigma_{p_{1}^{i} \mid p_{2}^{i}} \sqrt{\frac{\lambda_{0}}{\left[1-\frac{w_{i}}{\sum_{j=1}^{m} w_{j}}\right]\left[1-\frac{\delta_{p_{1}}}{k}\left(\frac{1+k \epsilon}{1+\epsilon}\right)\right]+\frac{\delta_{p_{1}}}{k}\left(\frac{1+k \epsilon}{1+\epsilon}\right)\left[1-c_{i}^{T} P_{\left[G, e_{m}\right]} c_{i}\right]}}
$$

In general, this MDB is of course smaller than the geometry-free case. This is due to the inclusion of the receiver-satellite geometry as represented by the projec-
Both of the previous MDBs depend on the receiversatellite geometry through the projector $P_{\left[G, e_{m}\right]}$. A useful approximation of the MDBs can be obtained if one 
makes use of the following property of projectors. Since the rank of a projector equals its trace, it follows from rank $P_{\left[G, e_{m}\right]}=4$ that the average value of the diagonal entries of the projector equals $\frac{4}{m}$. This average may then be used as an approximation to $c_{i}^{T} P_{\left[G, e_{m}\right]} c_{i}$.

\section{The cycle slip MDBs}

Cycle slips in the phase data are now considered. Here the cycle slip MDBs will be expressed in units of range rather than in units of cycles. For an $L_{1}$ phase-slip at epoch $l(1 \leq l \leq k)$ in the range to satellite $i \in\{1, \ldots, m\}$, the $c$-vector takes the form

$c=s_{l} \otimes d$ with $d=c_{3} \otimes d_{i}$

where the first $l-1$ entries of the vector $s_{l}$ equal zero and the last of its $k-l+1$ entries equals one. Since $s_{l}^{T} P_{e_{k}} s_{l}=\frac{N^{2}}{k}$, with the time-window $N=k-l+1$, the general expression for the cycle slip MDB follows from Eqs. (8), (18), (20) and (30) as

$$
\left|\nabla_{\phi_{1}}\right|=\sqrt{\frac{\lambda_{0} / N d^{T} Q^{-1} d}{1-\left(1-\frac{N}{k}\right) \frac{d^{T} Q^{-1} P_{M} d}{d^{T} Q^{-1} d}-\frac{N}{k} \frac{d^{T} Q^{-1} P_{[M, N]} d}{d^{T} Q^{-1} d}}}
$$

This expression still holds true for all three singlebaseline models. As before, its entries can be broken down into smaller parts if use is made of Eq. (21). For the roving-receiver case this gives

$$
\begin{aligned}
d^{T} Q^{-1} d & =d_{i}^{T} E^{-1} d_{i} / w_{i} \sigma_{\phi_{1}^{i} \mid \phi_{2}^{i}}^{2} \\
\frac{d^{T} Q^{-1} P_{M} d}{d^{T} Q^{-1} d} & =\delta_{\phi_{1}} \frac{1}{1+\epsilon} \frac{d_{i}^{T} E^{-1} P_{\bar{G}} d_{i}}{d_{i}^{T} E^{-1} d_{i}} \\
\frac{d^{T} Q^{-1} P_{[M, N]} d}{d^{T} Q^{-1} d} & =1
\end{aligned}
$$

with $\delta_{\phi_{1}}=1-\frac{\sigma_{\bar{\phi}}^{2}}{\sigma_{\phi_{2}}^{2}}$. As before, $\bar{G}$ should be replaced by $I_{m-1}$ to get the geometry-free result and $P_{M}$ should be set to zero to get the stationary-receiver result.

\subsection{Geometry-free model}

The cycle slip MDB of the geometry-free model follows from substituting Eqs. (26), (25) and (32) into Eq. (31) as

$$
\left|\nabla_{\phi_{1}^{i}}\right|=\frac{\sigma_{\phi_{1}^{i} \mid \phi_{2}^{i}}}{\sqrt{N}} \sqrt{\frac{\lambda_{0}}{\left[1-\frac{N}{k}\right]\left[1-\frac{w_{i}}{\sum_{j=1}^{m} w_{j}}\right]\left[1-\frac{\delta_{\phi_{1}}}{1+\epsilon}\right]}}
$$

This is the MDB for the $L_{1}$ phase observable. Interchanging $\phi_{1}$ and $\phi_{2}$ gives the MDB for the $L_{2}$ phase observable. The MDB depends on $\alpha_{o}, \gamma_{o}$, on the precision of code, on the precision of phase, on the number of epochs used and, through the weights $w_{i}$, on the number of satellites tracked. In contrast with the outlier MDB, since $N$ appears in the foregoing, the MDB now also depends on the moment the slip started to occur. Note that the MDB becomes infinite in case $N=k$. This reflects the situation that cycle slips cannot be found when they already commence with the first epoch $l=1$. In this case the slip cannot be separated from the corresponding phase ambiguity itself.

Also note, since the phase-code variance ratio $\epsilon$ is very small, that the precision of the code data has no significant impact on the value of the slip MDB. The slip MDB is therefore predominantly governed by the precision of the phase data. One can therefore expect to be able to detect sufficiently small cycle slips. However, it is important to realize that this only holds true for the dual-frequency case. In the single-frequency case we have $\delta_{\phi_{1}}=1$, which reduces Eq. (33) to

$$
\left|\nabla_{\phi_{1}^{i}}\right|=\frac{\sigma_{p_{1}^{i}}}{\sqrt{N}} \sqrt{\frac{(1+\epsilon) \lambda_{0}}{\left[1-\frac{N}{k}\right]\left[1-\frac{w_{i}}{\sum_{j=1}^{m} w_{j}}\right]}}
$$

This shows that in the single-frequency case it is not the high precision of the phase data that counts, but rather the relatively poor precision of the code data. This has an important impact on one's ability to detect cycle slips with the geometry-free model. Let us first consider the case $N=1$. It corresponds to the situation where the cycle slip occurs at the last epoch of the data set. In that case the smallest possible value of the MDB reads $\left|\nabla_{\phi_{1}^{i}}\right|=\sigma_{p_{1}^{i}} \sqrt{(1+\epsilon) \lambda_{0}}$. Hence in this case one cannot expect to find slips as small as one cycle. The only way to remedy this, and thus to pull the MDB down to smaller values, is to have $N>1$. But this implies that one will have to take a sufficient number of epochs into account after the slip occurred.

\subsection{Roving receiver}

For the case of the non-stationary baseline, where use is made of the receiver-satellite geometry, the cycle slip MDB follows from substituting Eqs. (26), (25) and (32) into Eq. (31) as

$$
\left|\nabla_{\phi_{1}^{i}}\right|=\frac{\sigma_{\phi_{1}^{i}} \mid \phi_{2}^{i}}{\sqrt{N}} \sqrt{\frac{\lambda_{0}}{\left[1-\frac{N}{k}\right]\left[\left(1-\frac{w_{i}}{\sum_{j=1}^{m} w_{j}}\right)\left(1-\frac{\delta_{\phi_{1}}}{1+\epsilon}\right)+\frac{\delta_{\phi_{1}}}{1+\epsilon}\left(1-c_{i}^{T} P_{\left[G, e_{m}\right]} c_{i}\right)\right]}}
$$


This MDB is smaller than that of Eq. (33), unless satellite redundancy is absent or $c_{i}$ lies in the range space of $\left[G, e_{m}\right]$. In both these cases the two MDBs will be equal. When they are not equal, it is of course the presence of the receiver-satellite geometry that makes the difference. But this is more so for the singlefrequency case than for the dual-frequency case. In the dual-frequency case a sufficiently small MDB is already reached without taking the receiver-satellite geometry into account, cf. Eq. (33). This is not so, however, in the single-frequency case, cf. Eq. (34). But this changes when the receiver-satellite geometry is included. It follows from Eq. (35) that then the MDB remains governed by the high precision of phase, even for the single-frequency case.

\subsection{Stationary receiver}

For the case of a stationary baseline, the projector $P_{M}$ can be set to zero. The corresponding cycle slip MDB follows then from substituting Eqs. (26), (25) and (32) into Eq. (31) as

$$
\left|\nabla_{\phi_{1}^{i}}\right|=\frac{\sigma_{\phi_{1}^{i}} \phi_{2}^{i}}{\sqrt{N}} \sqrt{\frac{\lambda_{0}}{\left[1-\frac{N}{k}\right]\left[1-\frac{w_{i}}{\sum_{j=1}^{m} w_{j}}\right]}}
$$

This MDB is again smaller than the previous one. Note that the MDB is independent of the receiver-satellite geometry. This is of course a consequence of the fact that we assumed matrix $\bar{G}_{k}$ to be time invariant. But since this is a good approximation for short time-spans, the above result does imply that the cycle slip MDB is not significantly influenced by the receiver-satellite geometry itself. Hence in the stationary case, it is not so much the geometric distribution of the satellites that counts, but more the number of satellites that are tracked.

\section{Summary}

In this contribution we derived closed-form expressions for the minimal detectable biases of outliers in the code data and of cycle slips in the phase data. The MDBs were given for three different single-baseline models: the geometry-free model, the roving-receiver geometrybased model and the stationary-receiver geometry-based model. They apply to both the single-frequency and the dual-frequency cases. The baselines were considered to be sufficiently short such that orbital uncertainties in the fixed orbits and residual ionospheric and tropospheric delays could be assumed absent. The stochastic model used permitted cross-correlation and allowed the use of different variances for the individual GPS observables. It also included the possibility to weigh the singledifference observables in dependence on which satellite is tracked.

It was shown that all outlier MDBs are insensitive to the precision of the phase data; they are predominantly governed by the precision of the code data. In the absence of cross-correlation and when the code data on the two frequencies are equally precise, the dual-frequencybased MDB acts as a single-frequency-based MDB using twice as many epochs.

In the single-frequency case, single-epoch-based outlier detection is not possible with the geometry-free model. It is possible, however, when use is made of the receiver-satellite geometry, provided satellite redundancy is present. This is true for the roving-variant and the stationary-variant, provided a critical configuration is absent. This configuration is one where all satellites but one are located on a cone having its symmetry axis perpendicular to the line-of-sight of the remaining satellite.

It was also shown that there is practically no difference between the MDBs of the roving and the stationary variants. Therefore constraining the baseline to be stationary does not improve one's ability to detect outliers in the code data.

Most, but not all cycle slip MDBs were shown to be governed by the high precision of the phase data. In these cases sufficiently small slips can be detected, even when using a time-window of $N=1$. The two exceptions occur in the single-frequency case. With the geometryfree model, it is then not the precision of phase, but the precision of code that governs the MDB. This implies that small slips cannot be found unless a sufficiently large time-window is used. In the presence of the critical configuration, this same situation occurs also when using the roving variant. It will not happen with the stationary variant, however, since it was shown that the corresponding MDB is not significantly influenced by the receiver-satellite geometry. In this case it is not the geometric distribution of the satellites that is important, but more the number of satellites tracked.

\section{References}

Baarda W (1967) Statistical concepts in geodesy. Netherlands Geodetic Commission, Publications on Geodesy, New Series, vol 2, no 4

Baarda W (1968) A testing procedure for use in geodetic networks. Netherlands Geodetic Commission, Publications on Geodesy, New Series, vol 2, no 5

Dedes G, Goad C (1994) Real-time cm-level GPS positioning of cutting blade and earth movement equipment. In: Proc ION-94, pp 587-594

Euler HJ, Goad C (1991) On optimal filtering of GPS dual-frequency observations without using orbit information. Bull Geod 65: $130-143$

Euler HJ, Hatch R (1994) Comparison of several AROF kinematic techniques. In: Proc ION-94. pp 363-370

Frei E, Beutler G (1990) Rapid static positioning based on the Fast Ambiguity Resolution Approach FARA: theory and first results. Manuscr Geod 15: 325-356

Hatch R (1982) The synergism of GPS code and carrier measurements. In: Proc 3rd Int Geod Symp Satellite Positioning, vol 2. pp 1213-1231

Hatch R (1991) Instantaneous ambiguity resolution. In: Kinematic systems in geodesy, surveying and remote sensing, IAG Symposium 127. Springer, Berlin Heidelberg New York, pp 299-308 
Hofmann-Wellenhof B, Lichtenegger BH, Collins J (1997) GPS theory and practice (4th edn) Springer, Vienna

Jin XX (1995) A recursive procedure for computation and quality control of GPS differential corrections. LGR Series, no 8, Delft Geodetic Computing Centre

Jin XX, Marel H van der, Jong CD de (1995) Computation and quality control of differential GPS corrections. In: Proc ION-95. pp 1071-1079

Jong CD de (1996) Real-time integrity monitoring of dual-frequency GPS observations from a single receiver. Acta Geod Geophys Hung 31: 37-46

Jong CD de (1997) Principles and applications of permanent GPS arrays, Delft University Press, Delft

Leick A (1995) GPS satellite surveying (2nd edn) Wiley, New York LGR staff (1982) The Delft approach for the design and computation of geodetic networks. In: Festschrift for W. Baarda. pp 202-274

Marel H van der (1990) Statistical testing and quality analysis of GPS networks. In: Proc 2nd Int Symp Precise positioning with the Global Positioning System. pp 935-949
Rao CR (1973) Linear statistical inference and its applications (2nd edn) Wiley, New York

Teunissen PJG (1985) Quality control in geodetic networks. In: Grafarend EW, Sanso F (eds) Optimization and design of geodetic networks. Springer, Berlin Heidelberg New York, Chapt 17

Teunissen PJG (1996) An analytical study of ambiguity decorrelation using dual-frequency code and carrier phase. J Geod 70: 515-528

Teunissen PJG, Kleusberg A (1996) GPS observation equations and positioning concepts. In: Kleusberg A, Teunissen PJG (eds) GPS for geodesy, Lecture notes in earth sciences, vol 60 . Springer, Berlin Heidelberg New York, Chapt 5

Teunissen PJG, Verhoef H (1996) A primer on network adjustment and quality control. ITB Press, Bandung, Indonesia

Teunissen PJG, Jonge PJ de, Tiberius CCJM (1995) The LAMBDA method for fast GPS surveying. In: Proc Int Symp GPS technology applications. pp 203-210

Tiberius, CCJM, de Jonge P (1995) Fast positioning using the LAMBDA method. In: Proc DSNS95. 